\title{
EXPERIMENTAL DESIGN AND BAYESIAN NETWORKS FOR ENHANCEMENT OF DELTA-ENDOTOXIN PRODUCTION BY BACILLUS THURINGIENSIS
}

\author{
KARIM ENNOURI ${ }^{1,2 *}$, RAYDA BEN AYED ${ }^{1,2}$, HANEN BEN HASSEN ${ }^{3}$, \\ MAURA MAZZARELLO ${ }^{4}$ and ENNIO OTTAVIANI ${ }^{4}$ \\ ${ }^{1}$ Laboratory of Probability and Statistics, Faculty of Sciences of Sfax, Sfax, Tunisia \\ ${ }^{2}$ Centre of Biotechnology of Sfax, Sfax, Tunisia \\ ${ }^{3}$ Physics-Mathematics and Applications, Faculty of Sciences of Sfax, Sfax, Tunisia \\ ${ }^{4}$ On AIR s.r.1., Genova, Italy
}

(Received: 13 December 2014; accepted: 6 June 2015)

\begin{abstract}
Bacillus thuringiensis (Bt) is a Gram-positive bacterium. The entomopathogenic activity of $\mathrm{Bt}$ is related to the existence of the crystal consisting of protoxins, also called delta-endotoxins. In order to optimize and explain the production of delta-endotoxins of Bacillus thuringiensis kurstaki, we studied seven medium components: soybean meal, starch, $\mathrm{KH}_{2} \mathrm{PO}_{4}, \mathrm{~K}_{2} \mathrm{HPO}_{4}, \mathrm{FeSO}_{4}, \mathrm{MnSO}_{4}$, and $\mathrm{MgSO}_{4}$ and their relationships with the concentration of delta-endotoxins using an experimental design (Plackett-Burman design) and Bayesian networks modelling. The effects of the ingredients of the culture medium on delta-endotoxins production were estimated. The developed model showed that different medium components are important for the Bacillus thuringiensis fermentation. The most important factors influenced the production of delta-endotoxins are $\mathrm{FeSO}_{4}, \mathrm{~K}_{2} \mathrm{HPO}_{4}$, starch and soybean meal. Indeed, it was found that soybean meal, $\mathrm{K}_{2} \mathrm{HPO}_{4}, \mathrm{KH}_{2} \mathrm{PO}_{4}$ and starch also showed positive effect on the delta-endotoxins production. However, $\mathrm{FeSO}_{4}$ and $\mathrm{MnSO}_{4}$ expressed opposite effect. The developed model, based on Bayesian techniques, can automatically learn emerging models in data to serve in the prediction of delta-endotoxins concentrations. The constructed model in the present study implies that experimental design (Plackett-Burman design) joined with Bayesian networks method could be used for identification of effect variables on delta-endotoxins variation.
\end{abstract}

Keywords: Bacillus thuringiensis, delta-endotoxins, Plackett-Burman, Bayesian networks

*Corresponding author; E-mail: karimennouri1@gmail.com 


\section{Introduction}

Bacillus thuringiensis (Bt) is a Gram-positive bacterium, producing parasporal crystals including delta-endotoxins responsible for its insecticidal potency [1] through sporulation. Upon ingestion by vulnerable insects, the crystals are dissolved in the midgut lumen. With the action of proteases, the endotoxins are changed into toxins that attach to receptors on the microvilli, causing damages to the epithelial midgut [2]. These proteins are highly toxic to a huge number of insect pests, safe to the environment, innocuous to non-target insect species. In general, conditions for the culture of $B$. thuringiensis are adjusted to have both high microbial biomass and delta-endotoxins concentration. A fermentation improvement plan can begin by estimating product yield as a solution to factors as activity of medium ingredients. Nutritional needs may be conducted either by the usual or mathematical approach. Mathematical methods present many advantages over usual methods being quick and decreases total number of trials [3]. In fact, Plackett-Burman design is considered as a section of a two-level factorial design and permits the probe of $n-1$ variables in at least $n$ experiments [4]. The Plackett-Burman design is suggested when more than five factors have to be studied. These designs are helpful for detecting great main effects, supposing the fact that all interactions are insignificant when compared with the few significant main effects.

Recently, Bayesian networks $(\mathrm{BN})$ have become an effective device for biological network reconstruction $[5,6,7]$. BNs constitute one of the most used formalisms for calculation and forecast under uncertainty. BNs offer a useful approach to illustrate the general dependency structure of a big number of variables, therefore removing the restriction of examining the relations between variables. The purpose of the present study is to develop a model that can automatically learn emerging models in data to serve in the prediction of delta-endotoxin concentrations.

\section{Materials and Methods}

\section{Microorganism}

The used strain BUPM5 of B. thuringiensis subsp. kurstaki is known by a high delta-endotoxin production [8]. The strain was maintained by streak inoculating Luria Broth (LB) nutrient plates $\left(\mathrm{g}^{-1}\right)$ : yeast extract 5, peptone $10, \mathrm{NaCl} 5$ and agar 15 , incubated at $30{ }^{\circ} \mathrm{C}$ for $24 \mathrm{~h}$ and stored at $48{ }^{\circ} \mathrm{C}$ for future use. 


\section{Inocula preparation method}

Inocula preparation was previously optimised based on delta-endotoxin production yields [9]. One isolated colony was dispensed in $3 \mathrm{ml}$ of LB medium and incubated overnight at $30^{\circ} \mathrm{C} .0 .5 \mathrm{ml}$ aliquots were used to inoculate $250 \mathrm{ml}$ shake flasks including $50 \mathrm{ml}$ of LB medium. After $6 \mathrm{~h}$ of incubation at $30^{\circ} \mathrm{C}$ at $200 \mathrm{rev} \mathrm{min}^{-1}$ in a rotary shaker (New Brunswick Scientific ${ }^{\mathrm{TM}}$, Edison, NJ, USA), the culture broth was used to inoculate the media. The O.D.600 was estimated using a SmartSpec ${ }^{\mathrm{TM}} 3000$ UV-spectrophotometer (Bio-Rad Laboratories). The culture broth was used to inoculate the culture media to begin with a primary optical density at $600 \mathrm{~nm}$ (O.D.600) of 0.15. Considering that an O.D.600 of 1 in B. thuringiensis bacterium is previously estimated to represent approximately $2 \times 10^{8} \mathrm{CFU} \mathrm{ml}^{-1}[10]$, the initial cell counts in the performed cultures are considered equal to approximately $3 \times 10^{-7} \mathrm{CFU} \mathrm{ml^{-1 }}$.

\section{Cultural conditions}

For delta-endotoxin production, B. thuringiensis strains were grown according to the medium compositions stated for each experiment (Table I). The $250 \mathrm{ml}$ shake flasks, containing $50 \mathrm{ml}$ of culture medium [11], were incubated for $72 \mathrm{~h}$ at $30{ }^{\circ} \mathrm{C}$ in a rotary shaker at $200 \mathrm{rev} \mathrm{min}^{-1}$. The obtained values are the means $( \pm \mathrm{SD})$ of three determinations of two different experiments.

Table I. Values of coded values used in factorial design $\left(\mathrm{g}^{-1}\right)$

\begin{tabular}{cccc}
\hline Nutrient code & Nutrient & Minimum value $(-1)$ & Maximum value $(+1)$ \\
\hline $\mathrm{X} 1$ & $\mathrm{KH}_{2} \mathrm{PO}_{4}$ & 0.5 & 1.5 \\
$\mathrm{X} 2$ & $\mathrm{~K}_{2} \mathrm{HPO}_{4}$ & 0.5 & 1.5 \\
$\mathrm{X} 3$ & $\mathrm{MgSO}_{4}$ & 0.1 & 0.5 \\
$\mathrm{X} 4$ & $\mathrm{MnSO}_{4}$ & 0 & 0.02 \\
$\mathrm{X} 5$ & $\mathrm{FeSO}_{4}$ & 0 & 0.02 \\
$\mathrm{X} 6$ & $\mathrm{Starch}$ & 25 & 35 \\
$\mathrm{X} 7$ & Soybean meal & 20 & 30 \\
\hline
\end{tabular}




\section{Delta-endotoxin determination}

Crystal proteins were dissolved before protein concentration assay as illustrated by Zouari et al. [10]. Crystal-spore pellets were washed twice with $1 \mathrm{M}$ of sodium chloride solution $(\mathrm{NaCl})$ and twice with distilled water. Subsequently, samples were incubated in $0.05 \mathrm{M} \mathrm{NaOH}(\mathrm{pH} 12.5)$ for $2 \mathrm{~h}$ at $30{ }^{\circ} \mathrm{C}$ in a rotary shaker $\left(200 \mathrm{rev} \mathrm{min}^{-1}\right)$. The soluble fractions were collected by centrifugation at $13000 \mathrm{rpm}$, for $10 \mathrm{~min}$. The supernatant including the alkali-soluble insecticidal proteins was used to estimate delta-endotoxins concentration by the Bradford method [12] using bovine serum albumin (BSA) as a standard. Samples were estimated at $595 \mathrm{~nm}$ after $10 \mathrm{~min}$.

\section{Screening of important nutrient components}

The significance of the different media components towards delta-endotoxin production was tested using Plackett-Burman experimental designs [4]. This technique is based upon the existence of Hadamard matrices, which are square matrices of order $N$ with entries at two levels, +1 and -1 . These matrices are orthogonal such that for each column the number of +1 is equivalent to the number of -1 . This statistical design is appropriate for screening the effect of a large number of factors in a trial and sufficient for the determination of main effects. With such experimental design, $N$ factors can be screened with only $N+1$ trials and screening up to 100 variables [13] is possible with the support of this technique. For screening aim, seven medium components have been tested using the Plackett-Burman design. The Plackett-Burman design was established on the first order model. The main effect was also estimated. Seven independent factors were evaluated in twelve trials and each factor was characterized by two levels: high and low concentrations. Statistical design analysis was carried out using the Minitab program package. The levels of independent and dependent variables evaluated in this study are listed in Table II [14]. The experimental designs, according to the Plackett-Burman method, are given in Table II.

\section{Bayesian networks modelling}

Bayesian networks (BNs) are a powerful framework for decision support under uncertain knowledge. They come out from artificial intelligence studies and constitute one of the most coherent techniques for the acquisition and the modelling of complex systems. They have been applied to a large range of prob- 
Table II. Study of variables on delta-endotoxin productions by Plackett-Burman design

\begin{tabular}{ccccccccc}
\hline Trial & $\mathrm{X} 1$ & $\mathrm{X} 2$ & $\mathrm{X} 3$ & $\mathrm{X} 4$ & $\mathrm{X} 5$ & $\mathrm{X} 6$ & $\mathrm{X} 7$ & $\mathrm{Y}$ \\
\hline 1 & +1 & +1 & -1 & +1 & -1 & -1 & -1 & $2130 \pm 90$ \\
2 & -1 & +1 & +1 & +1 & -1 & +1 & +1 & $3810 \pm 75$ \\
3 & -1 & -1 & +1 & +1 & +1 & -1 & +1 & $2350 \pm 100$ \\
4 & +1 & +1 & -1 & +1 & +1 & -1 & +1 & $3060 \pm 110$ \\
5 & +1 & -1 & +1 & +1 & -1 & +1 & -1 & $2300 \pm 85$ \\
6 & -1 & -1 & -1 & -1 & -1 & -1 & -1 & $1725 \pm 75$ \\
7 & +1 & +1 & +1 & -1 & +1 & +1 & -1 & $1975 \pm 80$ \\
8 & +1 & -1 & +1 & -1 & -1 & -1 & +1 & $3490 \pm 95$ \\
9 & +1 & -1 & -1 & -1 & +1 & +1 & +1 & $3500 \pm 105$ \\
10 & -1 & +1 & +1 & -1 & +1 & -1 & -1 & $1700 \pm 80$ \\
11 & -1 & -1 & -1 & +1 & +1 & +1 & -1 & $1240 \pm 60$ \\
12 & -1 & +1 & -1 & -1 & -1 & +1 & +1 & $3890 \pm 100$ \\
\hline
\end{tabular}

lems and eventually in biology. BNs are directed acyclic graphs composed by nodes (variables of the problem) and arcs that encode conditional probabilistic independencies between the nodes. These graphical models are very attractive for their aptitude to explain probabilistic interactions connecting variables. In fact, they have proven to capture causal relationships between variables and they can show excellent forecast accuracy even with relatively small sample data sizes $[15,16]$.

To achieve the mentioned objectives, Bayesian networks modelling was used. We considered 8 nodes ( 7 variables representing medium components and 1 node representing delta-endotoxins concentration). Since the data are discontinuous and experimental data produced using Plackett-Burman are limited, and it is well known that the application of BN requires a lot of data for the learning and testing procedures, our proposed methodology includes the following three different stages for building model: 1) Data normalization; 2) Construction of an undirected Gaussian graph; 3) Construction of Bayesian Network.

\section{Data normalization}

To normalize data from different experiences, a random sample with 116 data was created from experimental data (each new observation is the average of 30 observations). 


\section{Construction of an undirected Gaussian graph}

In order to define the different conditional interactions between variables (medium components) and delta-endotoxin concentration variable, a Gaussian graphical model $[17,18]$ was developed. The principle of the model is based on conditional independence. Indeed, from a full connected graph, an edge is removed between two nodes if the conditional independence between two variables is accepted. The graph obtained is called conditional independence graph. The used distribution of the variables is considered as Gaussian (implying normality of each variable). Therefore, two variables are independent only if the partial correlation coefficient is null [see (I)]. We consider graph consisting of $n$ nodes, each node $i$ is associated with a random variable $X_{i}$. It is assumed that the vector $X=\left(X_{1}, \ldots, X_{n}\right)$ is Gaussian with mean " $\mathrm{m}$ " and matrix empirical variance-covariance $\Sigma=\left(\sigma^{i j}\right)_{1 \leq i \leq j \leq n}$. We denote $\Sigma^{-1}=\left(\left(w^{i j}\right)\right)$ the precision matrix. The partial correlations can be defined as following:

$$
\left.\rho^{i j}=\operatorname{Corr}\left(X_{i}, X / X\right)\left(X_{i}, X_{j}\right)\right) \text { for } 1 \leq i \leq j \leq n
$$

Under the normality hypothesis, $X_{i}$ and $X_{j}$ are conditionally dependent only if $\rho^{i j} \neq 0$, it is know that $\rho^{i j}=-\frac{w^{i j}}{\sqrt{w^{i i} w^{j j}}}$. This formula estimates the partial correlations [19] and constructs a conditional independence graph.

\section{Construction of Bayesian Network}

A Bayesian network [20] is generated as following: two nodes $i$ and $j$ having a partial correlation are connected by a non-oriented edge. The orientation is determined by a heuristic method based on the following test: If $\mathrm{B}^{\mathrm{ij}}=\frac{w^{j j} \sigma^{i i}}{w^{i i} \sigma^{j j}}>1$, the arc is then oriented from $i$ to $j$ and if $\mathrm{B}^{\mathrm{ij}}=\frac{w^{j j} \sigma^{i i}}{w^{i i} \sigma^{j j}}<1$, the arc is then oriented from $j$ to $i$. The other edges with $\mathrm{B}^{\mathrm{ij}}=\frac{w^{j j} \sigma^{i i}}{w^{i i} \sigma^{j j}}=1$ remained undirected. The graph with all directed arcs constituted the Bayesian network. It is imperative to note that it does not necessarily include all nodes contained in the network [21]. The advantage of Bayesian network is to deduct all parent nodes (nutritional components) which are directly dependent on child nodes (delta-endotoxins concentration). Matlab program was used to analyze obtained data. 


\section{Results and Discussion}

\section{Medium component effects for delta-endotoxin production using Plackett-Burman design}

A total of seven components were screened through twelve experimental runs. The main effects of the components in the medium for delta-endotoxins production are presented in Figure 1. The soybean meal showed the maximum positive effect on delta-endotoxin production, followed by starch, $\mathrm{K}_{2} \mathrm{HPO}_{4}$ and $\mathrm{KH}_{2} \mathrm{PO}_{4}$. The effect of $\mathrm{FeSO}_{4}$ and yeast $\mathrm{MnSO}_{4}$ were negative indicating that these components are required in the medium for delta-endotoxins production but in lower concentration than the low level. $\mathrm{MgSO}_{4}$ had neutral effect on deltaendotoxin production.

Table III shows the results of regression (coefficient, standard error, $t$ and $p$-values) generated by applying experimental design technique. The significance of coefficients was estimated by Student's $t$-test and $p$-values. The higher the level of the $t$-value and the lower the $p$-value, the more significant is the coefficient [22]. All but $\mathrm{MgSO}_{4}$ were considered significant or marginally significant having an influence on the delta-endotoxin production on the 0.1 significance level (Table III). In our study, the soybean meal, starch, $\mathrm{FeSO}_{4}$ and $\mathrm{KH}_{2} \mathrm{PO}_{4}$ were the most significant nutrients for improvement of delta-endotoxin production by $B t$. However, $\mathrm{KH}_{2} \mathrm{PO}_{4}, \mathrm{MnSO}_{4}$ and $\mathrm{MgSO}_{4}$ were considered as insignificant. The use of high concentrations of $\mathrm{KH}_{2} \mathrm{PO}_{4}, \mathrm{~K}_{2} \mathrm{HPO}_{4}$, starch and soybean meal and exclusion of $\mathrm{FeSO}_{4}$ and $\mathrm{MnSO}_{4}$ increased the level of delta-endotoxin production. $\mathrm{MgSO}_{4}$ has no effect on production of delta-endotoxins. Besides, the coefficient of determination $\left(\mathrm{R}^{2}=98.42 \%\right)$ explains the high degree of collinearity between simulated and measured data. Likewise, $\mathrm{R}^{2}$ illustrates the proportion of the variance in measured data explained by the model. $\mathrm{R}^{2}$ ranges from 0 to 1 , with higher values indicating less error variance, and usually values greater than 0.5 are judged acceptable [23].

The statistical significance of the ratio, between the mean square variation (MS), due to regression, and the mean square residual error, was tested using analysis of variance (ANOVA) (Table IV). ANOVA is a statistical technique that subdivides the total variation of a set of data into component associated to specific sources of variation for the purpose of testing hypotheses for the modelled parameters. According to the ANOVA, the F-value was high, which indicates that variation on the response variable can be explained by the regression model. The associated $p$-value is used to estimate whether $\mathrm{F}$ is large enough to indicate statistical significance. A $p$-value (0.002) is lower than 0.01 , which indicates that the model is considered to be statistically significant [24]. 


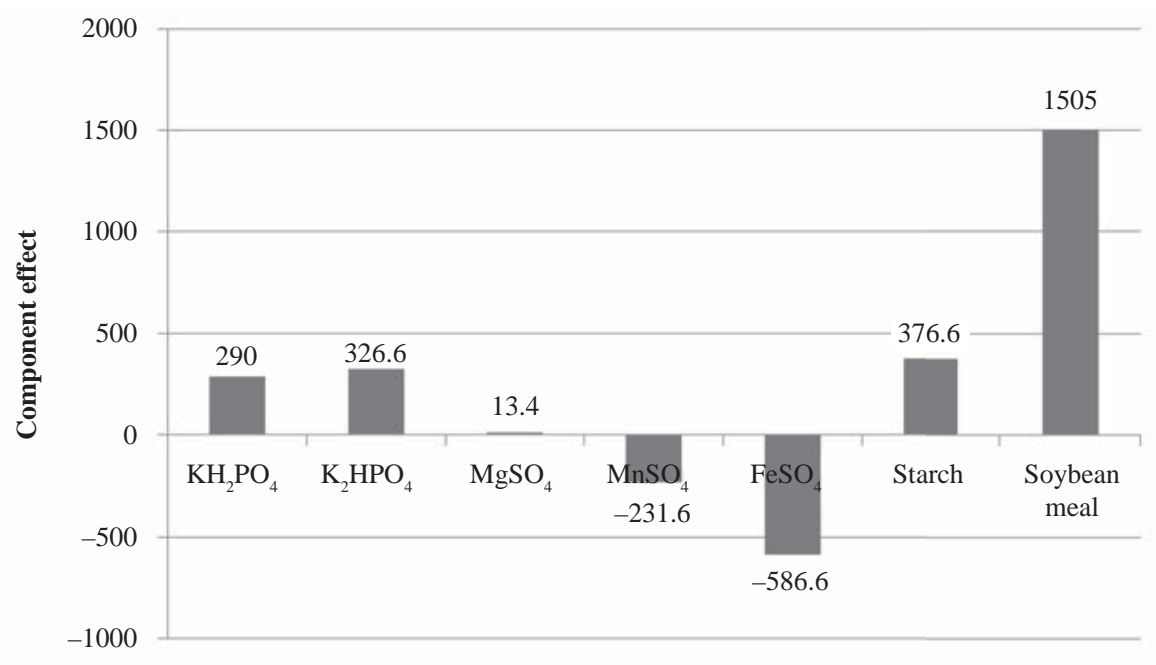

Medium component

Figure 1. Effect of media components on delta-endotoxins production in submerged culture

Table III. Estimated regression coefficients for delta-endotoxin production

\begin{tabular}{lcccc}
\hline Term & Coefficient & Standard Error & $t$ & $p$-value \\
\hline Constant & 2597.5 & 54.75 & 47.45 & 0.000 \\
$\mathrm{KH}_{2} \mathrm{PO}_{4}$ & 145.0 & 54.75 & 2.65 & 0.057 \\
$\mathrm{~K}_{2} \mathrm{HPO}_{4}$ & 163.3 & 54.75 & 2.98 & 0.041 \\
$\mathrm{MgSO}_{4}$ & 6.7 & 54.75 & 0.12 & 0.909 \\
$\mathrm{MnSO}_{4}$ & -115.8 & 54.75 & -2.12 & 0.102 \\
$\mathrm{FeSO}_{4}$ & -293.3 & 54.75 & -5.36 & 0.006 \\
$\mathrm{Starch}_{\text {Soybean meal }}$ & 188.3 & 54.75 & 3.44 & 0.026 \\
\hline
\end{tabular}

$R^{2}=98.42 \% ; R^{2}($ pred $)=85.82 \% ; R^{2}(\operatorname{adj})=95.67 \%$

The matching quality of the data obtained by the proposed model was evaluated by considering the correlation coefficient $\left(\mathrm{R}^{2}\right)$ between the experimental and modelled data. The statistical adjustment of those values generated an $\mathrm{R}^{2}=0.9836$, revealing that the model could not explain only $1.64 \%$ of the overall effect and showing that it is a robust statistical model. 
Table IV. Analysis of variance for delta-endotoxins

\begin{tabular}{lrrrrrr}
\hline Source & DF & Seq SS & Adj SS & Adj MS & F & $p$-value \\
\hline Main effects & 7 & 8.9478 & 8987217 & 1283888 & 35.70 & 0.002 \\
Residual error & 4 & 143858 & 143858 & 35965 & & \\
Total & 11 & 9131075 & & & \\
\hline
\end{tabular}

$\mathrm{R}^{2}=98.36 \% ; \mathrm{R}^{2}($ pred $)=85.23 \% ; \mathrm{R}^{2}(\operatorname{adj})=95.49 \%$

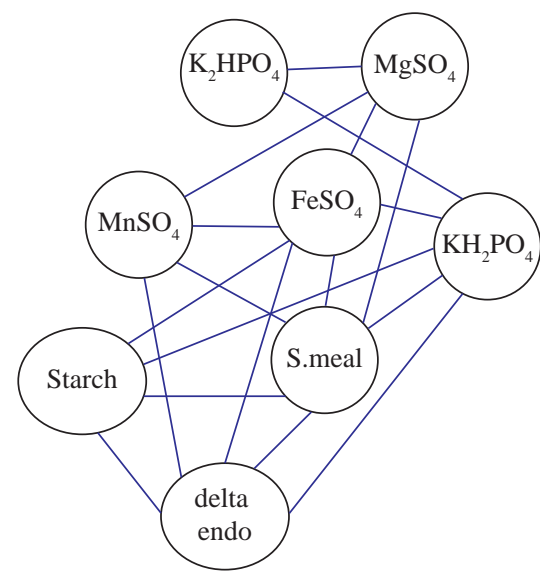

Figure 2. Gaussian graph representing conditional interaction between variables

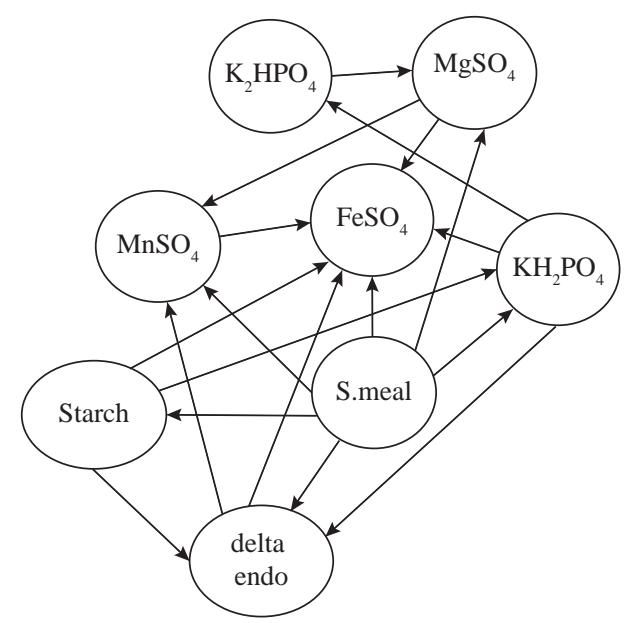

Figure 3. Bayesian network representing relationships between nutrients and delta-endotoxins

\section{Bayesian networks}

$\mathrm{BN}$ is a graph in which nodes represent variables and arcs represent dependencies among these variables. Usually, assigning a value to a variable determines the state of the variable. The obtained graphs for delta-endotoxins production are shown in Figures 2 and 3. The network contains 8 nodes: experimental variables, namely $\mathrm{FeSO}_{4}, \mathrm{MnSO}_{4}, \mathrm{MgSO}_{4}, \mathrm{KH}_{2} \mathrm{PO}_{4}, \mathrm{~K}_{2} \mathrm{HPO}_{4}$, soybean meal (S.meal) and starch, and the measured variable known as delta-endotoxins concentrations (delta endo).

Figure 2 illustrates an interconnection between different nodes in the network (with a minimum partial correlation of 0.45 ). The network also demonstrates that the output variable (delta endo) is strongly correlated with starch, 
soybean sulphate. These variables were the most influential on delta-endotoxin production.

The given result by the obtained Bayesian network (Figure 3) showed that the output node of delta-endotoxins was mainly determined by the "parents" identified as soybean meal, starch and potassium dihydrogen phosphorus. Other elements such as $\mathrm{FeSO}_{4}, \mathrm{MgSO}_{4}, \mathrm{MnSO}_{4}$ affected the production of the toxin, but indirectly. We also note that iron sulfate attracts the majority of nodes suggesting that $B t$ consumes iron in a complexed form. These observations are in line with several studies indicating high external validity. Indeed, soybean meal, the main source of nitrogen for $B t$, is considered as the most important nutrient during bacterial cell multiplication due to its participation in the construction of cellular proteins and nucleic acid synthesis. Nitrogen sources have direct control on crystal formation by stimulating the production of delta-endotoxin $[10,25]$. The degradation of starch, considered as a carbon source, produced acetic acid which is directly consumed by the bacterial cell and also the precursor of PHB production. Acetates are partly converted into intracellular PHB. Subsequently, acetates and PHB are assimilated in tricarboxylic acid cycle (TCA), a fundamental energy source for cell growth [26, 27]. According to Braun [28], the absence of manganese $(\mathrm{Mn})$ in the medium reduces the capacity of delta-endotoxin synthesis, suggesting the opposing direction of the arc. Similarly, Yang and Wang [29] deducted that the presence of $\mathrm{PO}_{4}{ }^{3-}$ in the culture medium is required to ensure better turnover of metabolic pathways. In fact, Gupta et al. [30] showed that phosphate has a role of regulator on the metabolite synthesis of $B t$ and is furthermore implicated in stimulation of delta-endotoxin production. This is confirmed by the Bayesian network indicating a causal relationship between potassium dihydrogen phosphate and delta-endotoxin production. On the other hand, the addition of ions such as $\mathrm{Fe}^{3+}, \mathrm{Mg}^{2+}, \mathrm{Cu}^{2+}, \mathrm{Co}^{+}$in the culture medium can improve the growth of $B t$ [31].

The effects of the parent nodes on output variable are estimated by multiple linear regression. ANOVA based on Bayesian method was carried out, the significance of each variable (parent) on delta-endotoxins production was determined using the Student's $t$-test and the accepted confidence level was 95\% (Table V). The regression results, $t$-values and $p$-values are given in Table V. Generally, factors having the larger $t$-value and smaller $p$-value were considered to be more significant in comparison to the factors whose $t$-value and $p$-value were different $[32,33]$. These results showed that the values of $\mathrm{KH}_{2} \mathrm{PO}_{4}$, starch and soybean meal were highly significant $(p<0.05)$. The high value of adjusted $\mathrm{R}$ square $\left(\mathrm{R}^{2}=0.941\right)$ suggests that $94.1 \%$ of the delta-endotoxins production 
Table V. Regression results for delta-endotoxins production

\begin{tabular}{lcccc}
\hline & Coefficient & SE & $t$ & $p$-value \\
\hline Constant & -3.2483 & 0.3359 & -9.6710 & $1.898 \times 10^{-16}$ \\
$\mathrm{KH}_{2} \mathrm{PO}_{4}$ & 0.4322 & 0.0337 & 12.8176 & $1.0625 \times 10^{-23}$ \\
Starch & 0.0648 & 0.0089 & 7.2799 & $4.9295 \times 10^{-11}$ \\
S.meal & 0.1378 & 0.0038 & 36.1672 & $1.3206 \times 10^{-63}$ \\
\hline
\end{tabular}

$\mathrm{R}^{2}=97 \% ; \mathrm{R}^{2}($ pred $)=93.94 \% ; \mathrm{R}^{2}(\operatorname{adj})=94.1 \%$

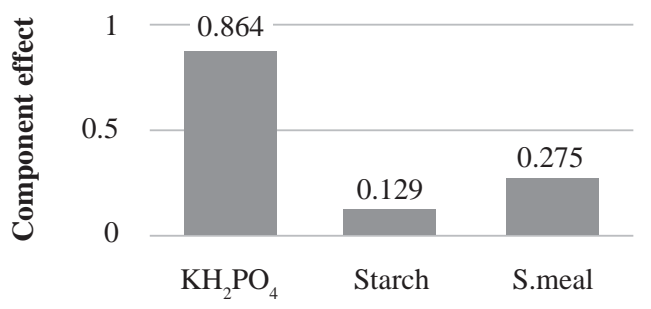

Main medium components

Figure 4. Effects of principal components on delta-endotoxins production

is mainly explained by these three variables. Besides, the regression model based on the Bayesian network displayed a higher value of predicted $\mathrm{R}$ squared $\left(\mathrm{R}^{2}(\right.$ pred $\left.)=93.94 \%\right)$, which indicates that the model fits well to the data and provides higher prediction power for future observations.

From Figure 4, we note an important influence of $\mathrm{KH}_{2} \mathrm{PO}_{4}$ on the deltaendotoxin production. Quantitatively, it is approximately estimated at 6 times greater than starch and 3 times higher than soybean meal.

\section{Conclusions}

The model developed in this study suggests that experimental design (Plackett-Burman design) coupled with Bayesian networks method could be employed for identification of effect variables on response variation. However it has been demonstrated only for very simple systems. A wider range of parameters of nutritional conditions should be examined in order to establish the impact on response variables, such as delta-endotoxins in our case. 


\title{
Acknowledgement
}

This study was supported by grants from the "Tunisian Ministry of Higher Education, Scientific Research and Technology".

\section{Conflict of Interest}

\author{
No conflict of interest.
}

\section{References}

1. Schnepf, E., Crickmore, N., Van Rie, J., Lereclus, D., Baum, J., Feitelson, J., Zeigler, D. R., Dean, D. H.: Bacillus thuringiensis and its pesticidal crystal proteins. Microbiol Mol Biol Rev 62, 772-806 (1998).

2. Ruiz, L. M., Segura, C., Trujillo, J., Orduz, S.: In vivo binding of the Cry $11 \mathrm{Bb}$ toxin of Bacillus thuringiensis subsp. medellin to the midgut of mosquito larvae. Mem Inst Oswaldo Cruz 99, 73-79 (2004).

3. El-sersy, N. A., Abou-Elela, G. M.: Antagonistic effect of marine Nocardia brasiliensis against the fish pathogen Vibrio damsela: Application of Plackett-Burman experimental design to evaluate factors affecting the production of the antibacterial agent. IJOO 1, 141-150 (2006).

4. Plackett, R. L., Burman, J. P.: The design of optimum multifactorial experiments. Biometrika 33, 305-325 (1946).

5. Grzegorczyk, M., Husmeier, D., Edwards, K. D., Ghazal, P., Millar, A. J.: Modelling nonstationary gene regulatory processes with a non-homogeneous Bayesian network and the allocation sampler. Bioinformatics 24, 2071-2078 (2008).

6. Radhakrishnan, N., Scutari, M., Lèbre, S.: Bayesian Networks in R with Applications in Systems Biology. Springer, New York, Heidelberg, Dordrecht, London, 2013, 157 pages.

7. Scutari, M., Denis, J. B.: Bayesian Networks with Examples in R. Chapman \& Hall/CRC, 2014, 225 pages.

8. Ennouri, K., Ben Khedher, S., Jaoua, S., Zouari, N.: Correlation between delta-endotoxin and proteolytic activities produced by Bacillus thuringiensis var. kurstaki growing in an economic production medium. Biocontrol Sci Techn 23, 756-767 (2013).

9. Ghribi, D., Zouari, N., Jaoua, S.: Improvement of bioinsecticides production through mutagenesis of Bacillus thuringiensis by u.v. and nitrous acid affecting metabolic pathways and/or delta-endotoxin synthesis. J Appl Microbiol 97, 338-346 (2004).

10. Zouari, N., Dhouib, A., Ellouz, R., Jaoua, S.: Nutritional requirements of a Bacillus thuringiensis subsp. kurstaki strain and use of gruel hydrolysate for the formulation of a new medium for delta-endotoxin production. Appl Biochem Biotech 69, 41-52 (1998).

11. Ghribi, D., Zouari, N., Trigui, W., Jaoua, S.: Use of sea water as salts source in starch- and soya bean-based media, for the production of Bacillus thuringiensis bioinsecticides. Process Biochem 42, 374-378 (2007). 
12. Bradford, M. A.: Rapid and sensitive method for the quantitation of microgram quantities of protein utilizing the principle of protein-Dye binding. Anal Biochem 72, 248-254 (1976).

13. Castro, P. M. L., Hayter, P. M., Ison, A. P., Bull, A. T.: Application of a statistical design to the optimization of culture medium for recombinant interferon-gamma production by Chinese hamster ovary cells. Appl Microbiol Biotechnol 38, 84-90 (1992).

14. Ennouri, K., Ben Hassen, H., Ben Khedher, S., Zouari, N.: Concomitant production of delta-endotoxins and proteases of Bacillus thuringiensis subsp. kurstaki in a low-cost medium: Effect of medium components. Acta Biol Szeged 57, 13-19 (2013).

15. Bensi, M., Der Kiureghian, A., Straub, D.: Efficient Bayesian network modeling of systems. Reliab Eng Syst Safe 112, 200-213 (2013).

16. Benson, M.: Bayesian Networks Handbook. M L Books International, New Delhi, 2015, 126 pages.

17. Whittaker, J.: Graphical Models in Applied Multivariate Statistics. Wiley, Chichester, 1990, 462 pages.

18. Lauritzen, S. L.: Graphical Models Oxford University Press, Oxford, 1996, 312 pages.

19. Marrelec, G., Krainik, A., Duffau, H., Pélégrini-Isaac, M., Lehéricy, S., Doyon, J., Benali, H.: Partial correlation for functional brain interactivity investigation in functional MRI. Neuroimage 32, 228-237 (2006).

20. Pearl, J.: Causality: Models Reasoning, and Inference. Cambridge University Press, Cambridge, 2000, 400 pages.

21. Opgen-Rhein, R., Strimmer, K.: From correlation to causation networks: A simple approximate learning algorithm and its application to high-dimensional plant gene expression data. BMC Syst Biol 1, 1-37 (2007).

22. Khuri, A. I., Cornell, J. A.: Response Surfaces: Designs and Analyses, Marcel Dekker, New York, 1987, 405 pages.

23. Santhi, C., Arnold, J. G., Williams, J. R., Dugas, W. A., Srinivasan, R., Hauck, L. M.: Validation of the SWAT model on a large river basin with point and nonpoint sources. J Am Water Resour Assoc 37, 1169-1188 (2001).

24. Kim, H. M., Kim, J. G., Cho, J. D., Hong, J. W.: Optimization and characterization of UV-curable adhesives for optical communications by response surface methodology. Polym Test 22, 899-906 (2003).

25. Icgen, Y., Icgen, B., Ozcengiz, G.: Regulation of crystal protein biosynthesis by Bacillus thuringiensis: Effects of carbon and nitrogen sources. Res Microbiol 9, 605-609 (2002).

26. Liu, W. M., Bajpai, R., Bihari, V.: High-density cultivation of sporeformers. Ann NY Acad Sci 721, 310-325 (199 4).

27. Feng, K. C., Liu, B. L., Chan, H. S., Tzeng, Y. M.: Morphology of a spectrum of parasporal endotoxin crystals from cultures of Bacillus thuringiensis ssp. kurstaki isolate A3-4. World J Microb Biot 17, 119-123 (2001).

28. Braun, S.: Production of Bacillus thuringiensis insecticides for experimental uses. In: Bioassays of Entomopathologic Microbes and Nematodes, CABI Publishing, Cambridge, 2000, pp. 49-72.

29. Yang, X., Wang, S. S.: Development of Bacillus thuringiensis fermentation and process control from a practical perspective. Biotechnol Appl Biochem 28, 95-98 (1998). 
30. Gupta, R., Beg, Q. K., Khan, S., Chauhan, B.: An overview on fermentation, downstream processing and properties of microbial alkaline proteases. Appl Microbiol Biot 60, 381395 (2002).

31. Bernhard, K., Utz, R.: Production of Bacillus thuringiensis insecticides for experimental and commercial uses. In: Entwistle, P. F., Cory, J. S., Bailey, M. J., Higgs, S. (Eds.) Bacillus thuringiensis, An Environmental Biopesticide: Theory and Practice. John Wiley \& Sons, Chichester, UK, 1993, pp. 59-68.

32. Prakash, O., Talat, M., Hasan, S. H., Pandey, R. K.: Factorial design for the optimization of enzymatic detection of cadmium in aqueous solution using immobilized urease from vegetable waste. Bioresour Technol 99, 7565-7652 (2008).

33. Liu, H. L., Chiou, Y. R.: Optimal decolorization efficiency of Reactive Red 239 by $\mathrm{UV} / \mathrm{TiO}_{2}$ photocatalytic process coupled with response surface methodology. Chem Eng J 112, 173-179 (2005). 